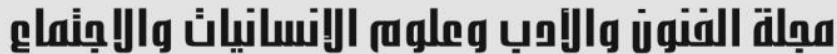

Journal of Arts, Literature, Humanities and Social Sciences www.jalhss.com

\section{واقع تفعيل إدارة المعرفة في مجتمع المعلومات}

شادية سعدالله عبدالله

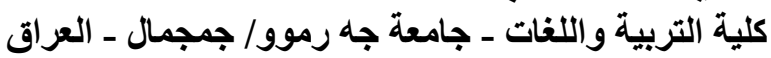
الايميل: shadya.sadulla@charmouniversity.org

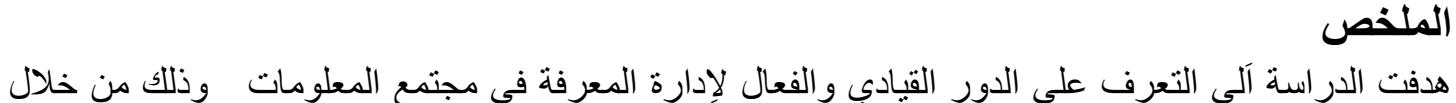

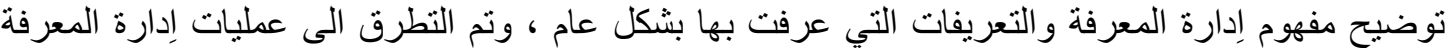

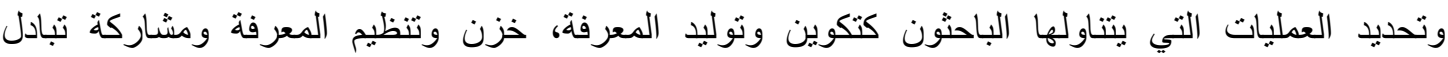
المعلومات، فضلا عن العناصر الأساسية لإدارة المعرفة، وتناولت الدراسة كيفية تطبيق إدارة المعرفة في

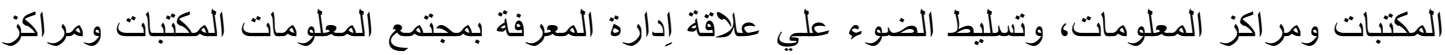
المعلومات نموذجا. وقد حاولت الدراسة ألاجابة علي تساؤلات مشكلة الدراسة و الموضح في الاطار العام للار اسة و أستخدام في ذلك

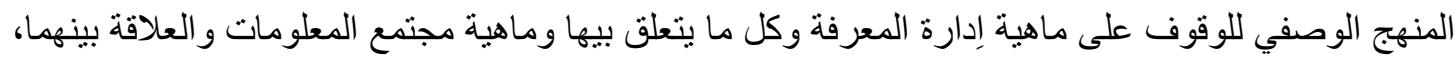
و أستخدمت الباحثة المصادر الورقية فضلا عن المصادر الالكترونية كمصادر معتمدة عليها فضلا فيلا عن استخدام

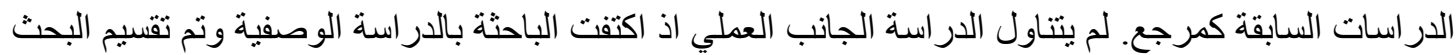
الى ثلاثة مباحث المبحث الاول تناولت مفهوم إدارة المعرفة والمبحث الثاني تناول مفهوم مجتمع المعلومات الثات الثات وكيفية تطبيقها في المكتبات ومر اكز المعلومات، اما المبحث الثالث والثات والاخير فتنتاولت مدى علات علاقة مفهوم إدارة

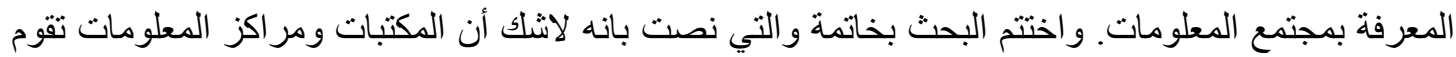

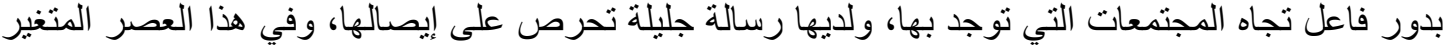

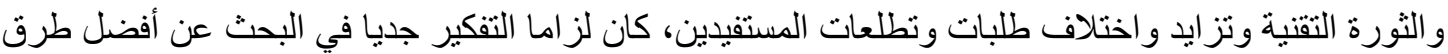
تحسين الأداء والتطوير المستمر في المكتبات ومر اكز المعلومات لآيصال المعلومة بأسر ع وقت ممكن و أقل جهد

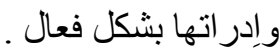

الكلمات المفتاحية: دور إدارة المعرفة، تكنولوجيا المعلومات في إيجاد الميزة التنافسية، مجتمع المستفيدين،

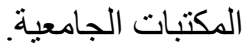




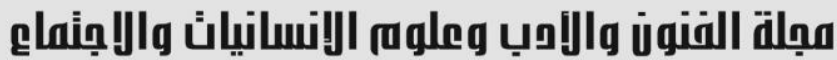 \\ Journal of Arts, Literature, Humanities and Social Sciences

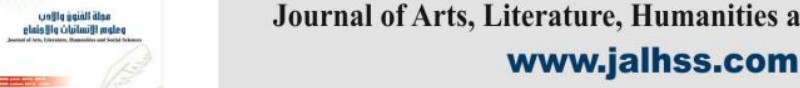 \\ Volume (58) September 2020

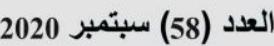

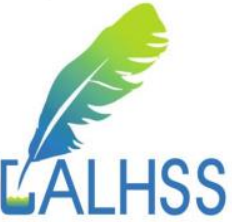 \\ The State of Activating Knowledge Management in Information Community
}

\author{
Shadya Sadullah Abdullah \\ College of Education and Languages -Charmouniversity/Chamchamal - Iraq \\ Email: Shadya.sadulla@charmouniversity.org
}

\begin{abstract}
The study aimed to identify the leading and effective role of knowledge management in the realm of information by clarifying the concept of knowledge management and its definitions in general. Moreover, the study touched upon the processes of knowledge management and the processes that researchers deal with such as forming, generating, storing and organizing, sharing and exchanging it information exchange besides the basic elements of knowledge management. The study dealt with how to apply knowledge management in libraries and information centers, and shed light on the relationship of knowledge management with the community of information like, libraries and information centers as a model.

The study has answered to answer a number of questions of presented in the study problem using a descriptive approach to identify knowledge management and everything related to it and to recognize the concept of information community and the relationship between them.

The researcher used paper references as well as reliable electronic references of the previous studies. The study did not deal with the practical side, as the researcher was satisfied with the descriptive study.

The research was divided into three sections. The first deals with the concept of knowledge management. The second deals with the concept of the information community and how it is applied in libraries and information centers. As for the final section, it covers the extent of the relationship of the concept of knowledge management with the information community. The research ends with a conclusion which states that there is no doubt that libraries and information centers play an active role towards the societies in which they are located, and they have a great message that they are keen to deliver. It also stresses that in this changing era and the technical revolution and the increasing of different demands and aspirations of the beneficiaries; it was necessary to seriously think about the search for the best ways to improve the performance of libraries and develop information centers to effectively deliver information as quickly as possible with least effort and management.
\end{abstract}

Keywords: The role of knowledge management, information technology in creating a competitive advantage, the community of users, University libraries. 


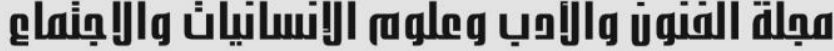

Journal of Arts, Literature, Humanities and Social Sciences www.jalhss.com

1. المشكلة الدار استة: تتمحور مشكلة الدراسة حول التساؤلات التالية :

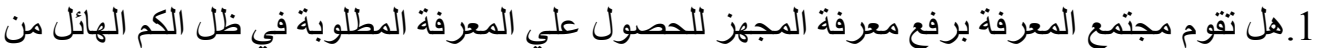

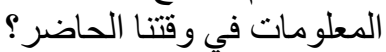

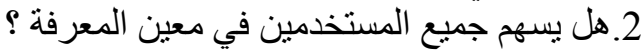

3. هل الككتبات ومر اكز المعلومات تتصل بمدى عريض من المصنادر للحصول على أفضل المعلومات عن

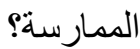
4. هل تعيد المكتبات ومر اكز المعلومات البنية المادية من أجل تسهيل المشاركة في المعرفة؟ 2. أهمية البحث: يستمد البحث اهميته من المنظلقات التالية: 1. أ تطوير التكنولوجيات الحيثة في مجال المعلومات.

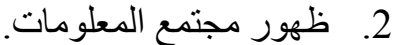
3. 3. بروز العولمة وحتمية التعامل معها. 4. انعكاسات التطورات التكنولوجية على المكتبات الجامعية في وظائفها وخدماتها.

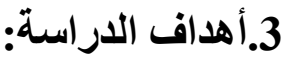

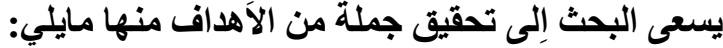

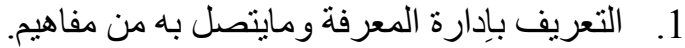
2.

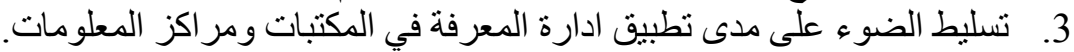

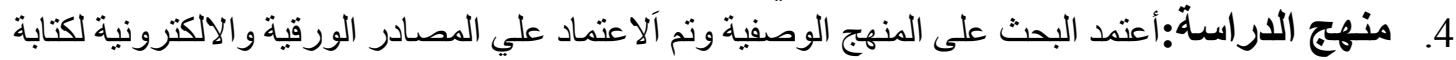
(البحث.

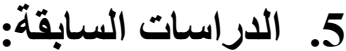

- - الدراسة الأولي : الدكتبات في مجتمع المعلومات، عبد اللطيف صوفي ،2003.

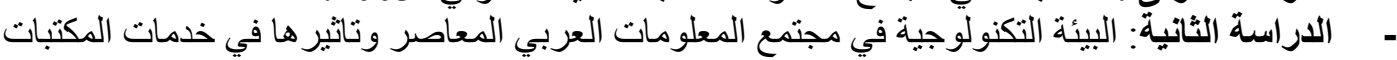
- - الدراسة الثالتة: دور المكتبات في مجتمع المعلومات ،روس شيمون. - - الاراسة الرابعة : مجتمع المعلومات و اثثره في الدكتبات الجامعية: مدينة قسنطينة نموذجا، مر اد كريم .2008،

\section{المبحث الاول

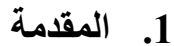
يؤكد الباحثون في حقل المعرفة على ضرورة فهم العلاقة بين (البيانات والمعلومات و المعرفة ) حيث يرتبط

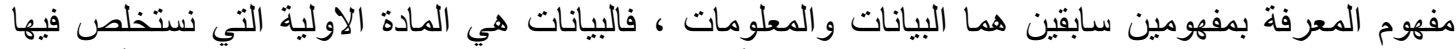

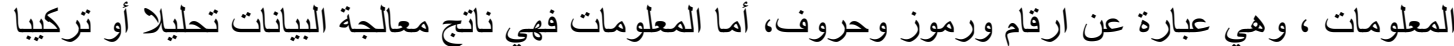

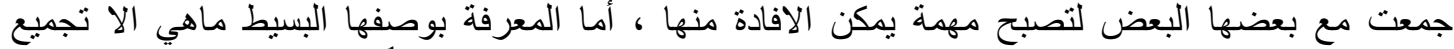

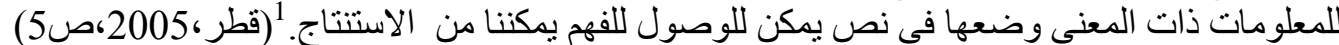

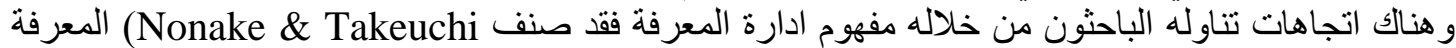

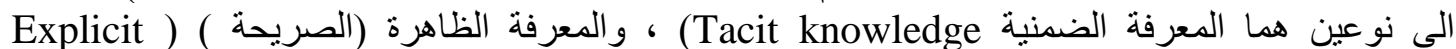

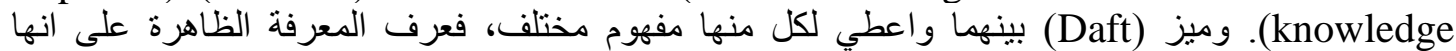

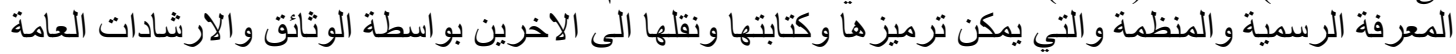

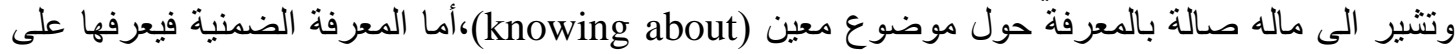




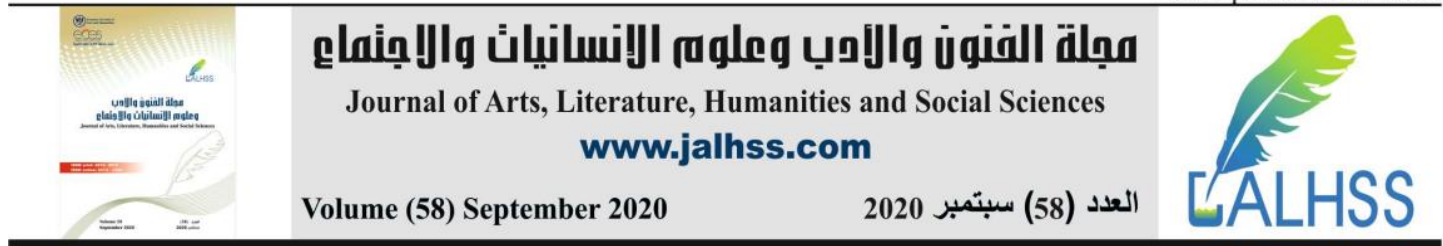

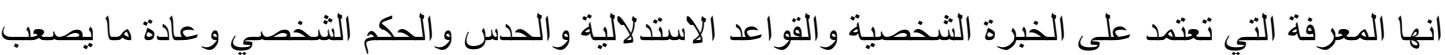

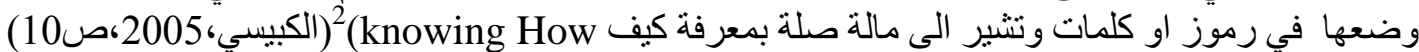

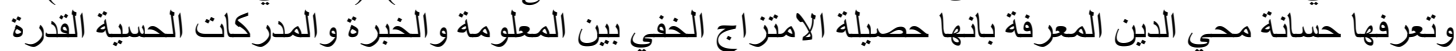

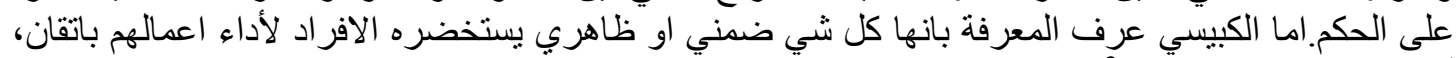

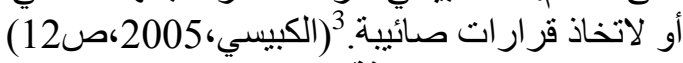

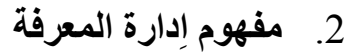

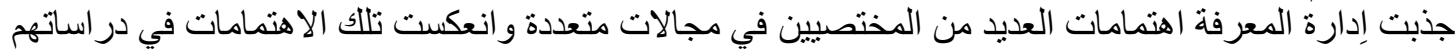

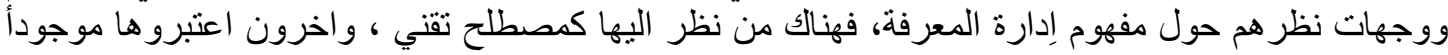
غير ملموس، و البعض الاخر ركز على ملى مفهوم

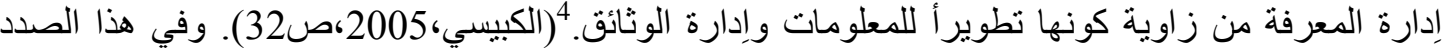

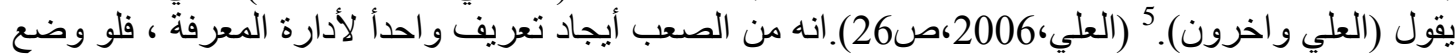

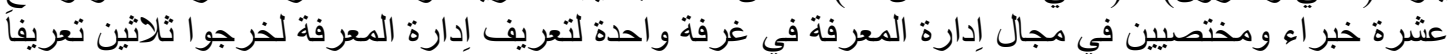

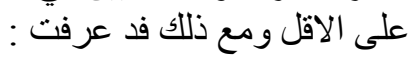

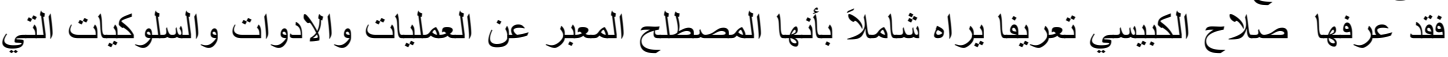

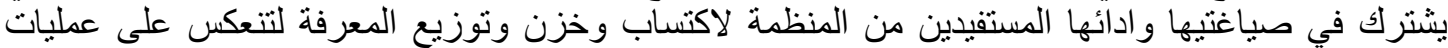

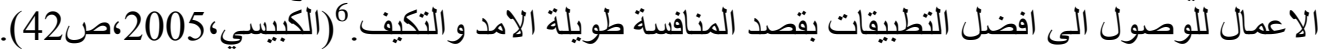

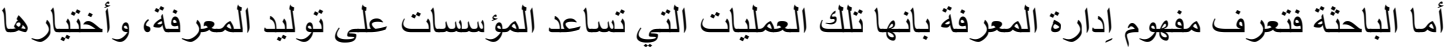

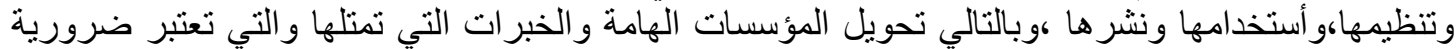

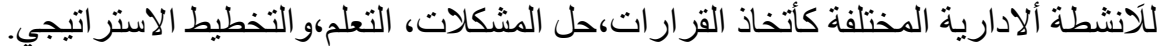

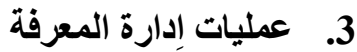

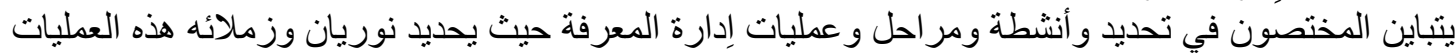

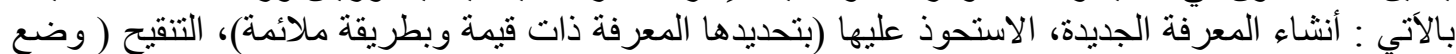

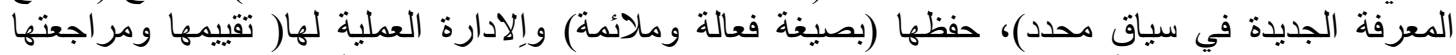
وتوضيح صلتها ودقتها)، أما ديفيد سكام فقد حدد عمليات إدارة المعرفة تحديدا تفصليا أوسع من نوريان التهان وزملائه

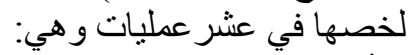

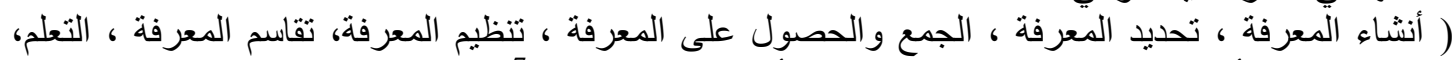

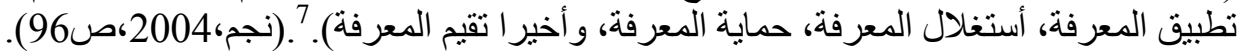

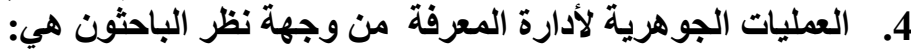

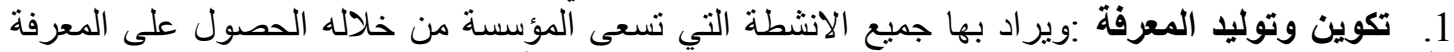

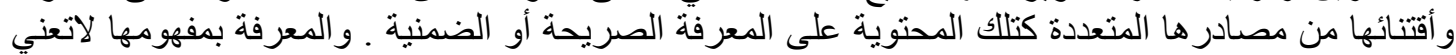

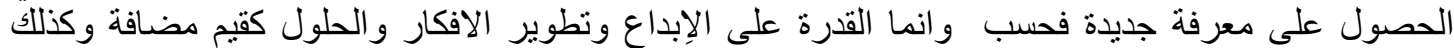

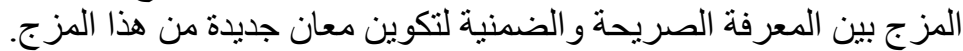

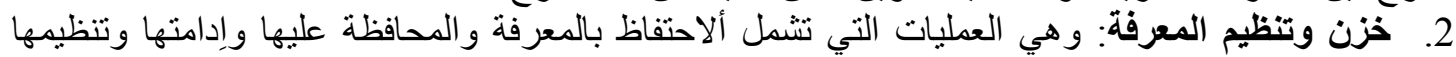

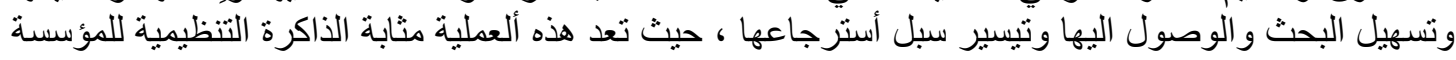
وفي هذه العميلة ييرز دور استخدام تقنية المعلومات في العديد من مر احل هذه العملية . 


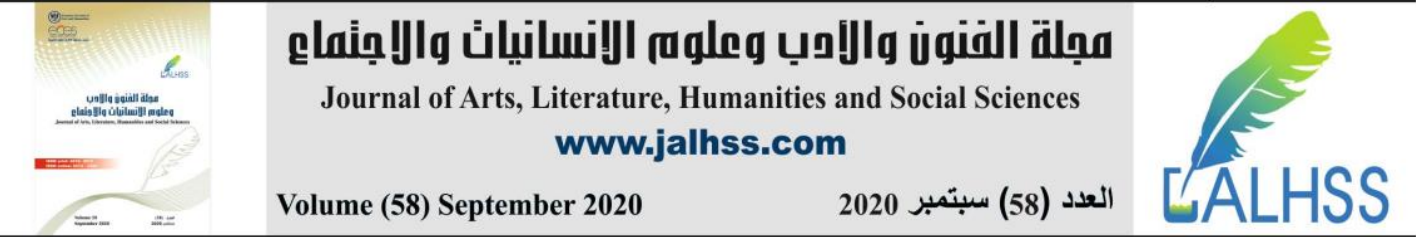

3. نقل ومشاركة المعرفة: تعني نقل ومشاركة المعرفة بين افر اد المؤسسة حيث يتم توزيع المعرفة الضمنية

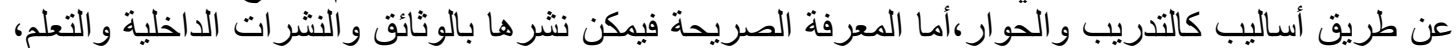

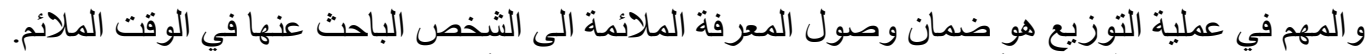

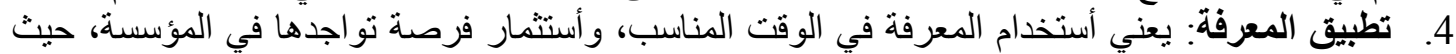

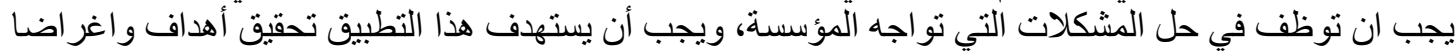

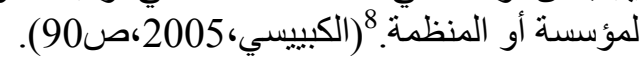

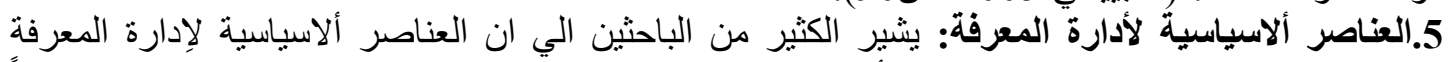

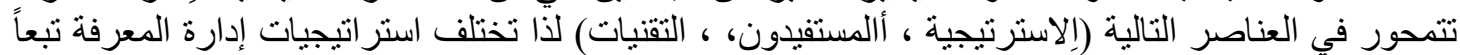

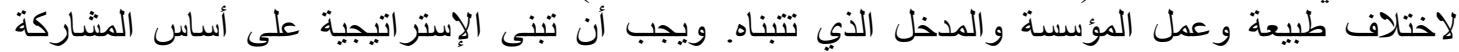

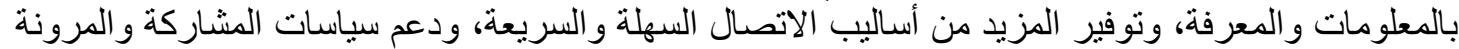

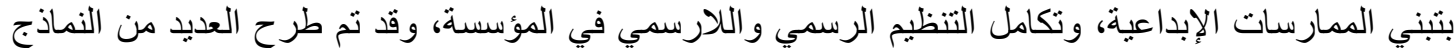
لإدارة المعرفة، و التي تركزت الإبدة على بناء هياكل التعلم الفردي و التنظيمي وبناء نقافة التئة المشاركة المعرفية. من بينها

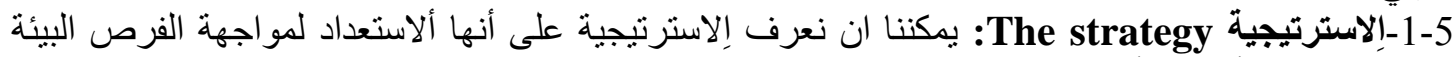

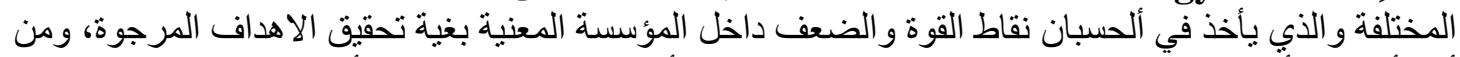

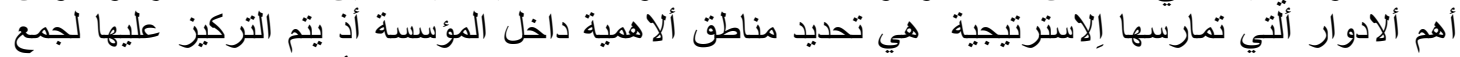

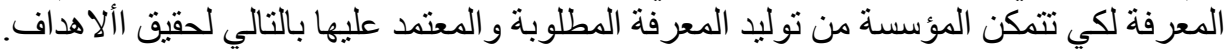

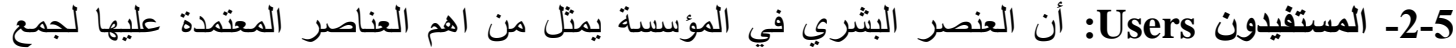

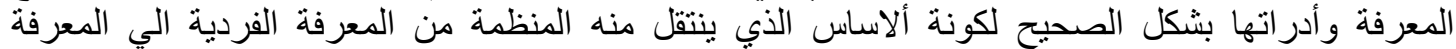

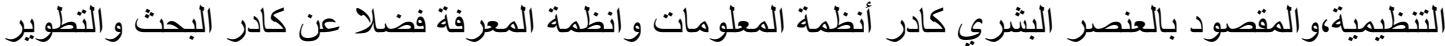

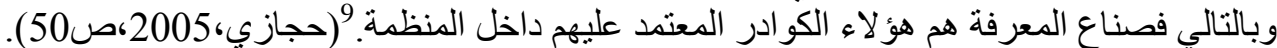

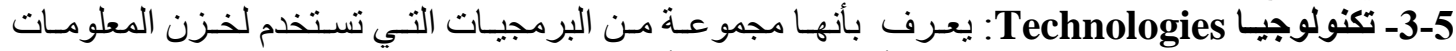

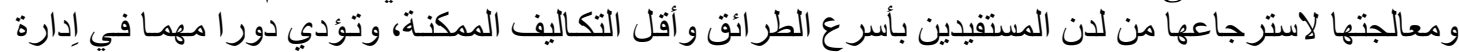

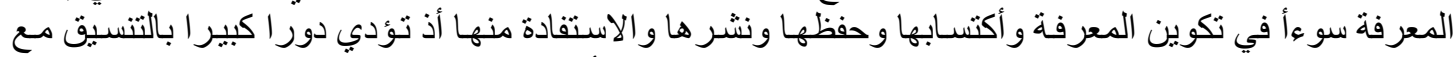

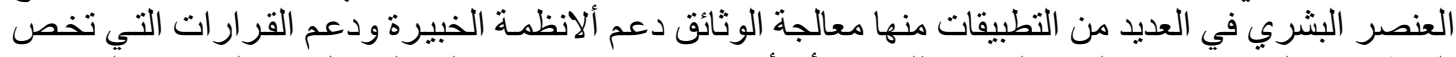

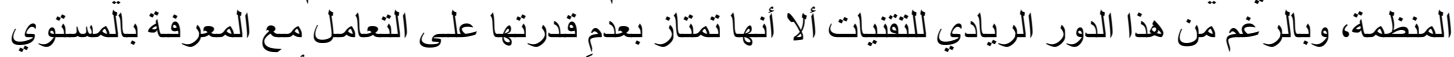

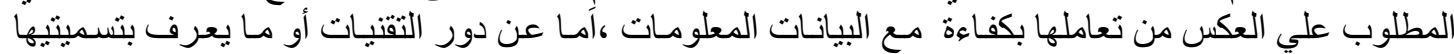

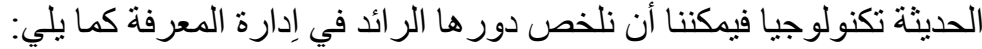
- أسهمت التكنولوجيا في تنميط وتسهيل وتسريع وتبسيط كل عمليات إدارة المعرفة فئة من توليد وتحليل وخزن

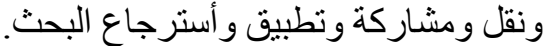
- أسهمت في تعزيز أمكانية السيطرة على المئل المعرفة الموجودة و التي جعلت منها عملية سهلة وذات تكلفة اقل وميسرة بنفس الوقت للأستخدام. - ساهمت في تهئية بيئة مناسبة مع تفاعل المو ارد البشرية لخلق معرفة جديدة. 10 (الكبيسي،2005،ص95).

5.تطبيق إدارة المعرفة في المكتبات ومراكز المعلومات:

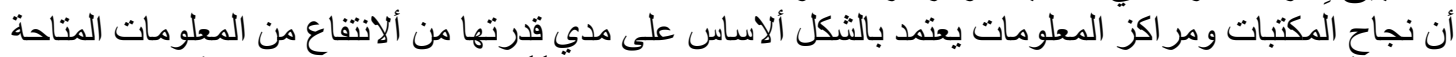

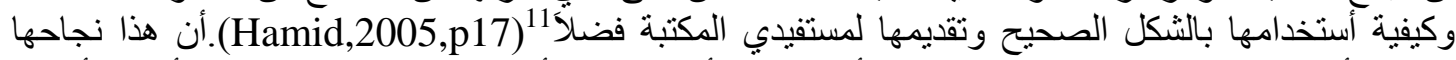

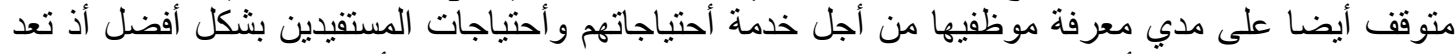

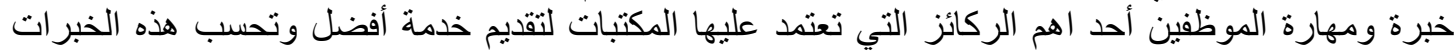




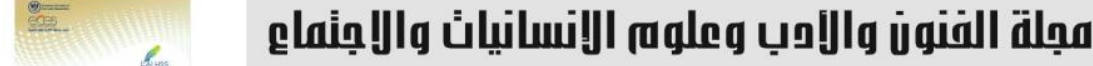 \\ Journal of Arts, Literature, Humanities and Social Sciences www.jalhss.com

و المهارة بالنهاية من ضمن ممتلكات المكتبة الثمينة و التي لايمكن التفريط بيها. //www.white-clouds.com/iclc)

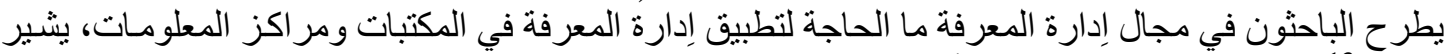

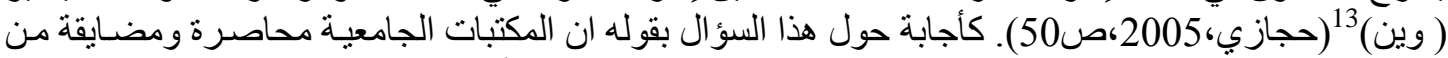

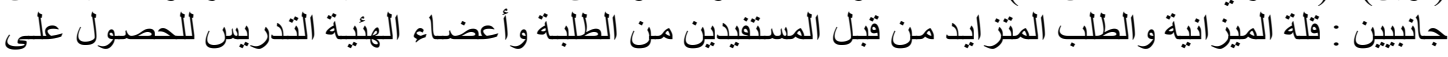

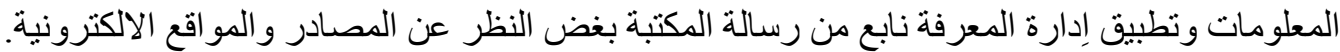

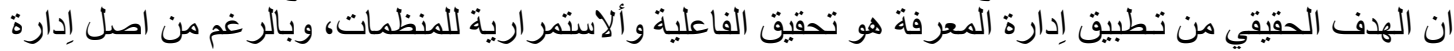

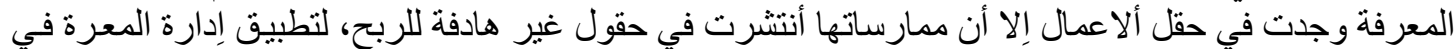

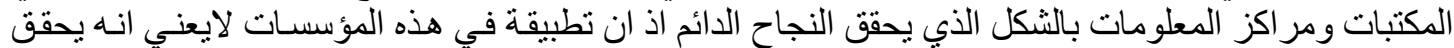

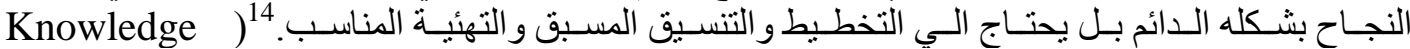
Management Section. IFLA Home Page. Available at http//www.ifla.org)

في السنوات الماضية اهتمت الاتحاد الدولي لجمعيات المكتبات (افلا) بِادارة المعرفة وتطبيقاتها في المكتبات

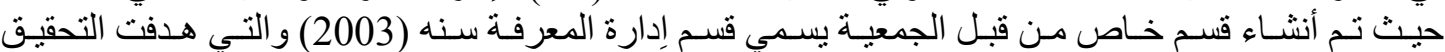

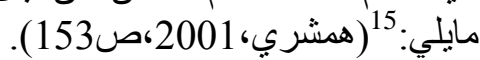

- - مانشاء إطار دولي يمكن من خلاله تحقيق اتصال دولي مهني وفهم طبيعة و أهمية إدارة المعرفة. - - السعى لمعرفة تطبيقات إدارة المعرفة في المكتبات ومر اكز المعلومات و التعرف على على أهم تطور اتهه في هذا

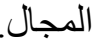

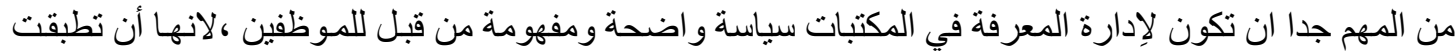

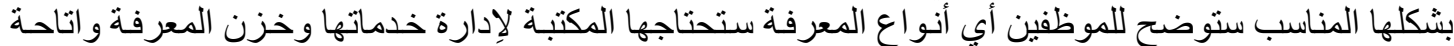

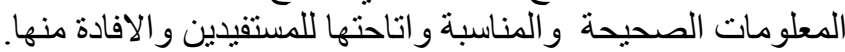

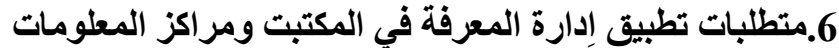

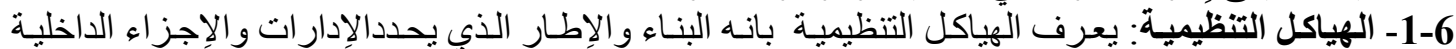

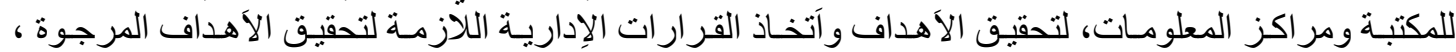

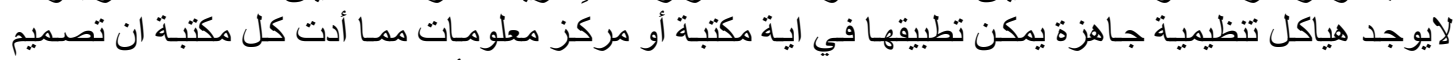

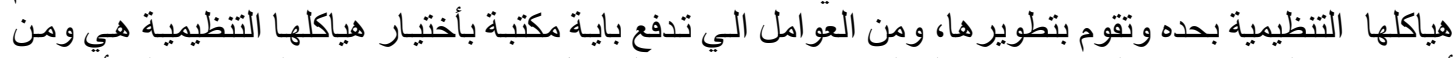

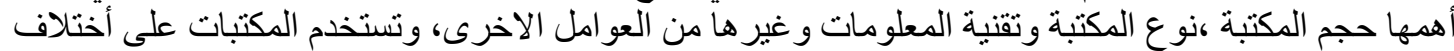

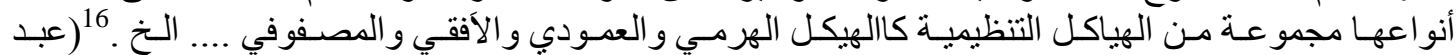

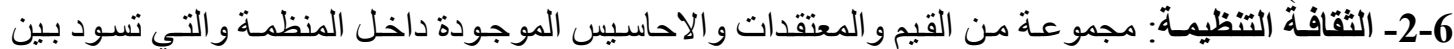

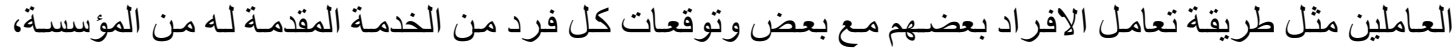

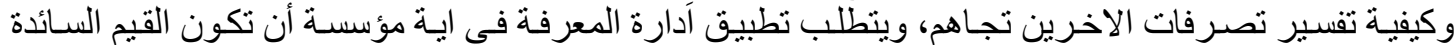

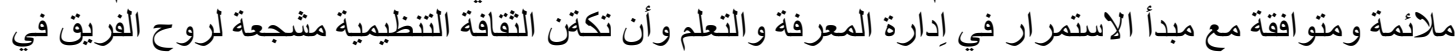

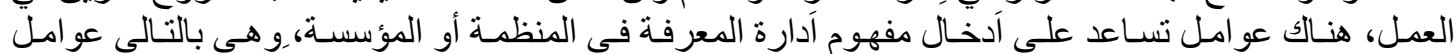
أيجابية لإدارة المعرفة في المؤسسات ومن بينها المكتبات ومر اكز آلمعلومات ومن هذه المنات العو امل الثنقافة التني تحث 


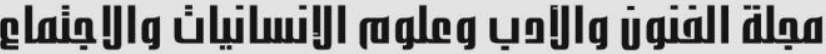

Journal of Arts, Literature, Humanities and Social Sciences www.jalhss.com

وتتجع على العمل لابروح الفريق الواحد وتساعد على تبادل الأفكار الآيجابية بين افر اد الفريق او طاقم العمل

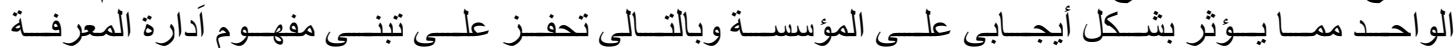
7.

3-7-7 القيادة ومدير المعرفة: أن مفهوم القيادة عنصر مهم في تبني وتطوير وتطبيق إدارة المعرفة ، اذئ يعد القائد

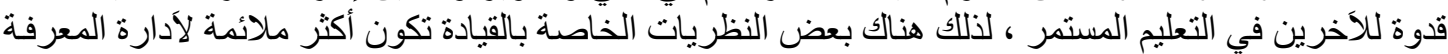

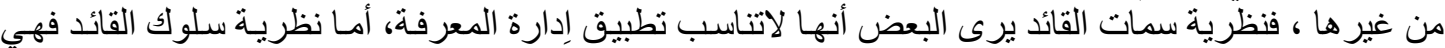

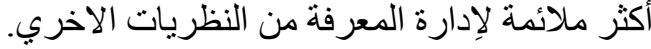

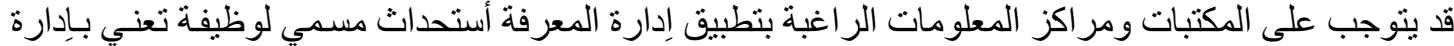

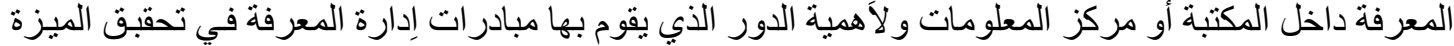

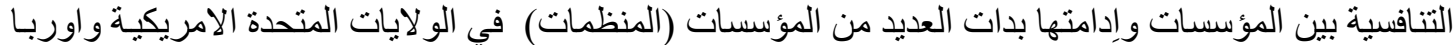

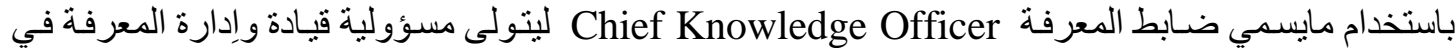
هذه المنظمات، لذا فان دور قائد المعرفة ينحصر في المهام التالية : - الدفاع عن المعرفة اذ تفرض التغيرات الطويلة الامد وسلوكيات الافر اد العاملة المتعلقة بالعمل الدفاع عنها بشكلا أيجابيا . - - تصميم وتنفيذ البنبة التحتية للمعرفة ومر اقتيها بما يشمل في ذلك المكتبات وقو اعد المعرفة.

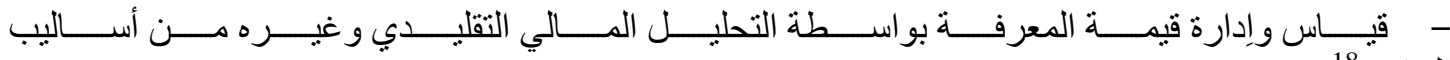

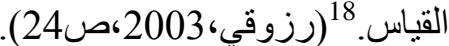

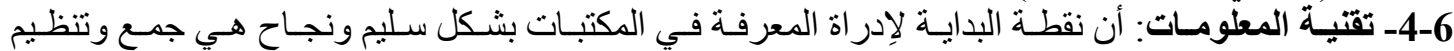

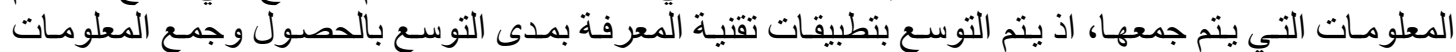

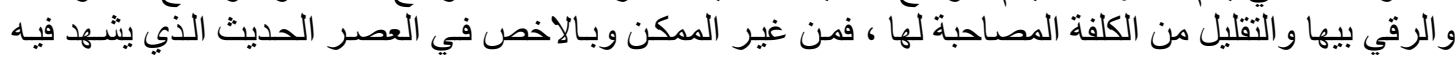

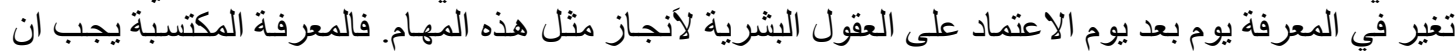

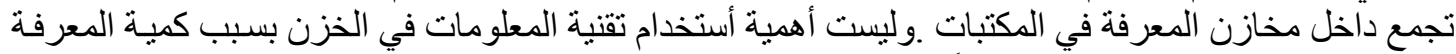

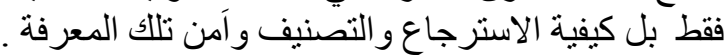

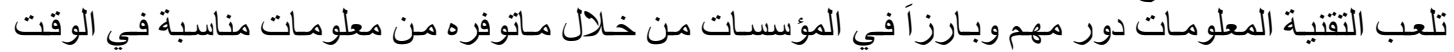

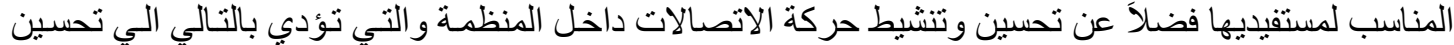

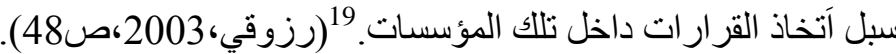




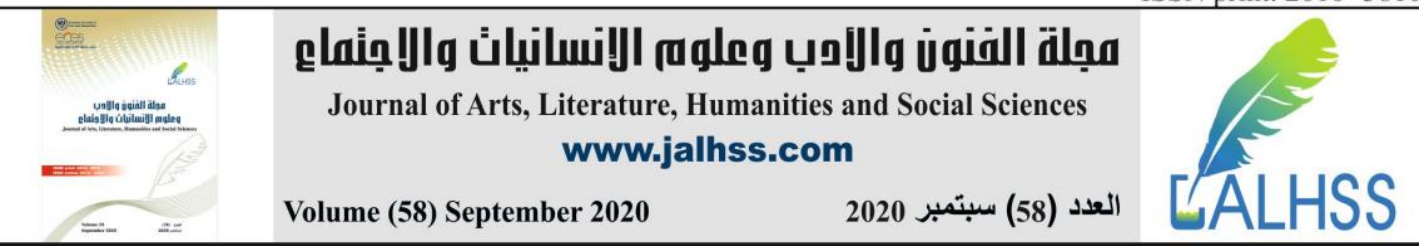

مخطط يوضح منطلبات تطبيق إدارة المعرفة في المكتبات ومر اكز المعلومات

الهياكل التنظيمية

تقتية المعلومات

\section{القيادة ومدير المعرفة}

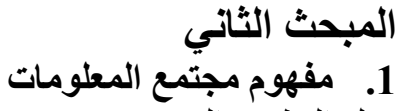

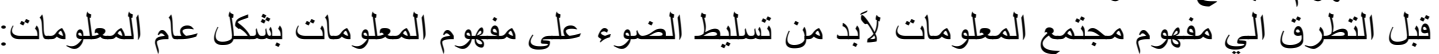

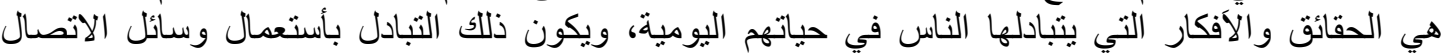

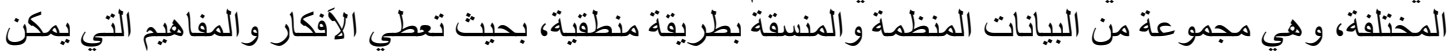

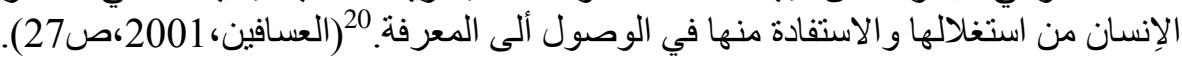

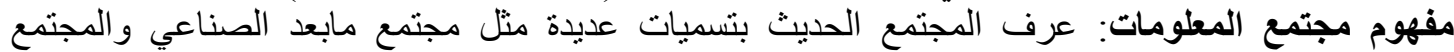

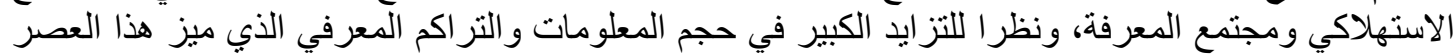

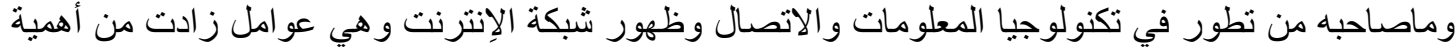

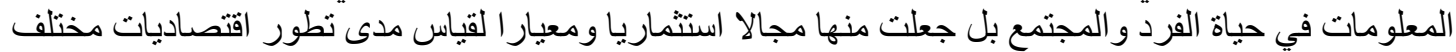

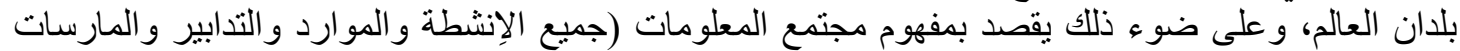

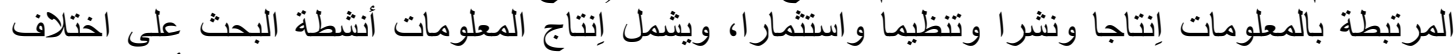

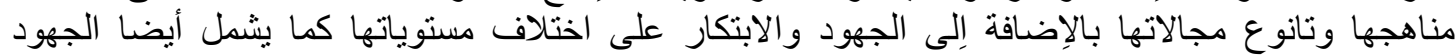

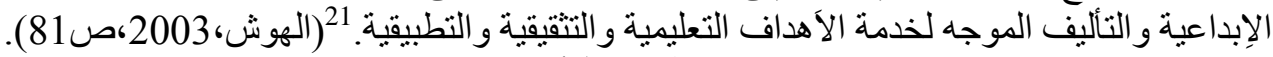

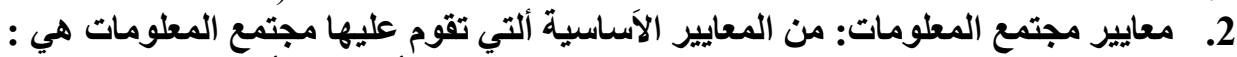

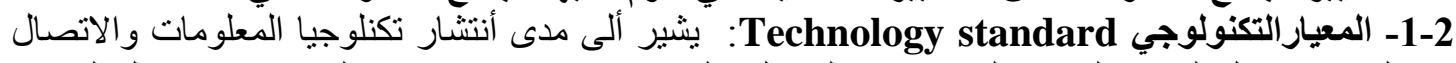
في كل مكان داخل المدن و القرى و المؤسسات و المنازل و المدارس ومدى استخدامها و التحكم فيها من قبل الجهات التهات المستخدمة لها. 2-2-2 المعيارالاجتماعي Social standard: يقصد بيها فهم الآفر اد وإدر اكهم لاَهمية المعلومات وتقدر هم له

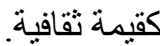
الافئة-2-2 المعيارالاقتصادي Economic standard : أى المستوى الاقتصادي للأفر اد، دخلهم المادي ومواردهم

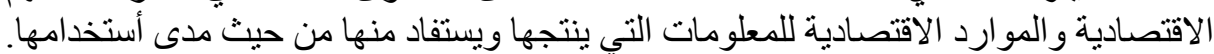

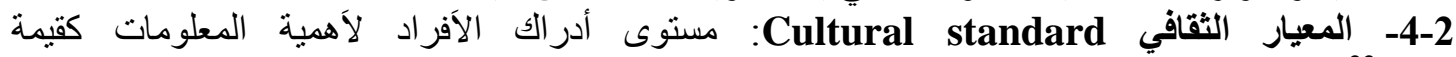

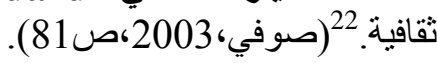




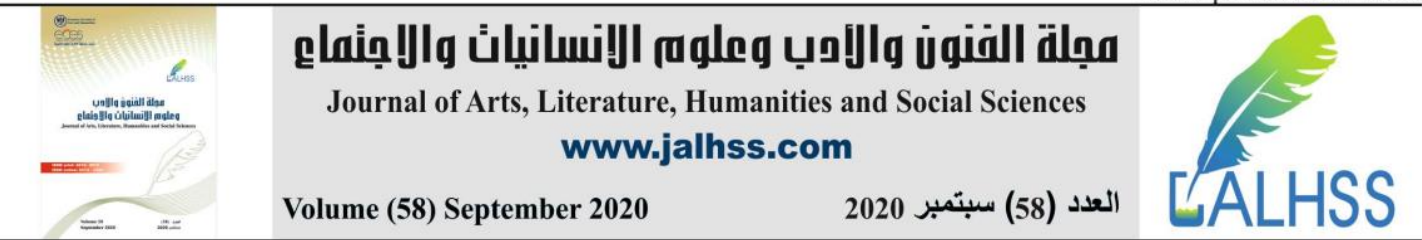

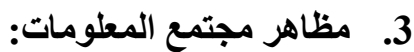

1-3-3 الحكومة الإكترونية The Electronic Government: مفهوم الحكومة الإلكترونية تقوم على اساس

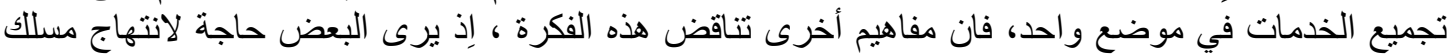

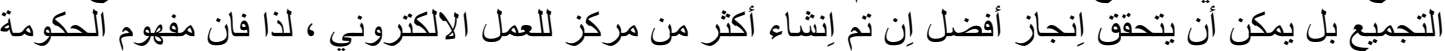

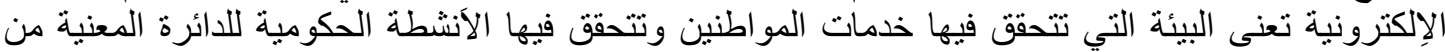

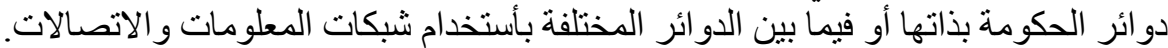

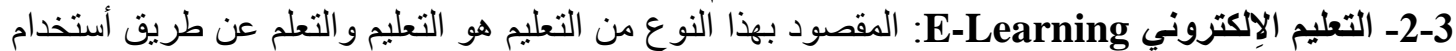

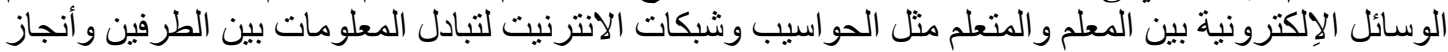

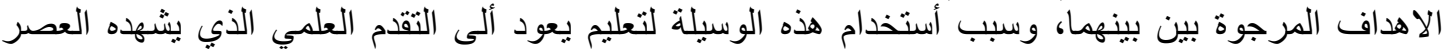

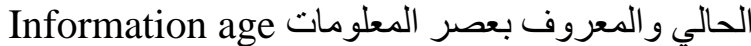

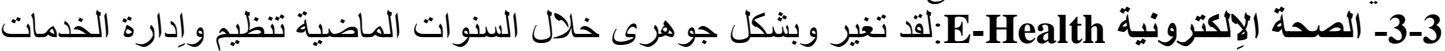

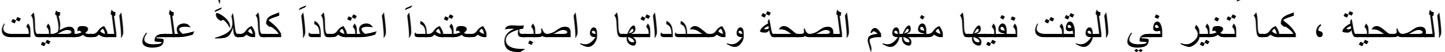

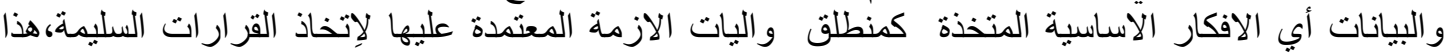

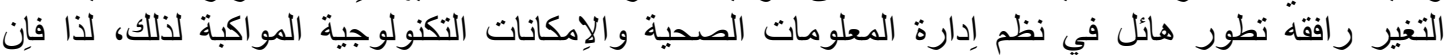

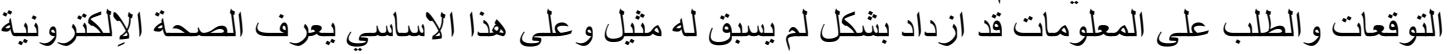

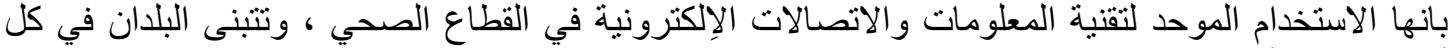

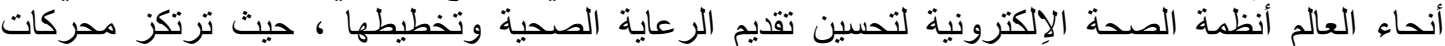

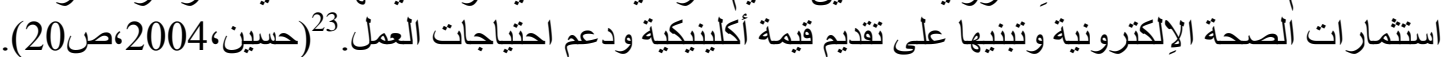
-4-3 النشر الإكتروني Electronic publishing: يعد النشر الإلكتروني تقنية حديثة يستخدم وسائل الاتصال مختلفة كالأنترنيت وشبكات المعلومات فضلا عن الوسائط المتعددة ذات الكثافة التخزينية العالية لنقل

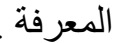

يعرف بانها عملية إصدار عمل مكتوب بالوسائل الإلكترونية وخاصة الحاسوب سواء مباثرة أو من خلال

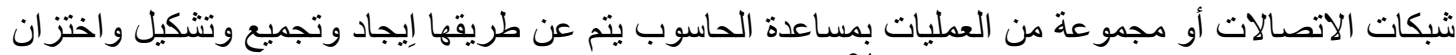

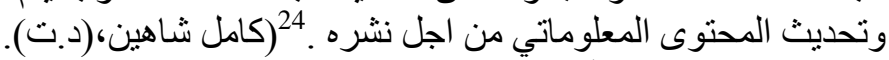

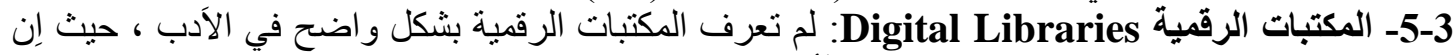

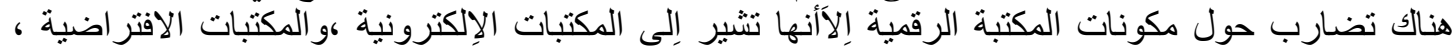

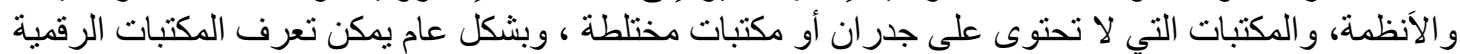

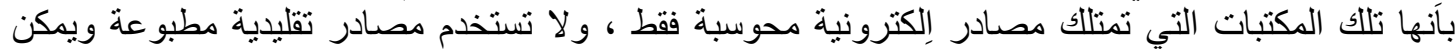

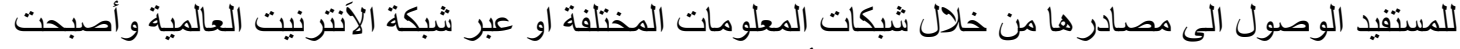

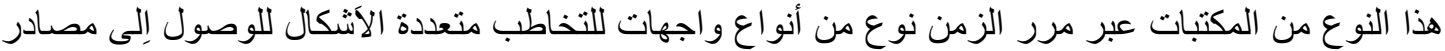

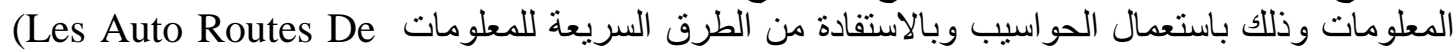
(Anne, التي توفر ها شبكات المعلومات المختلفة وشبكات الأنترنيت العالمية. 2014,p33). 


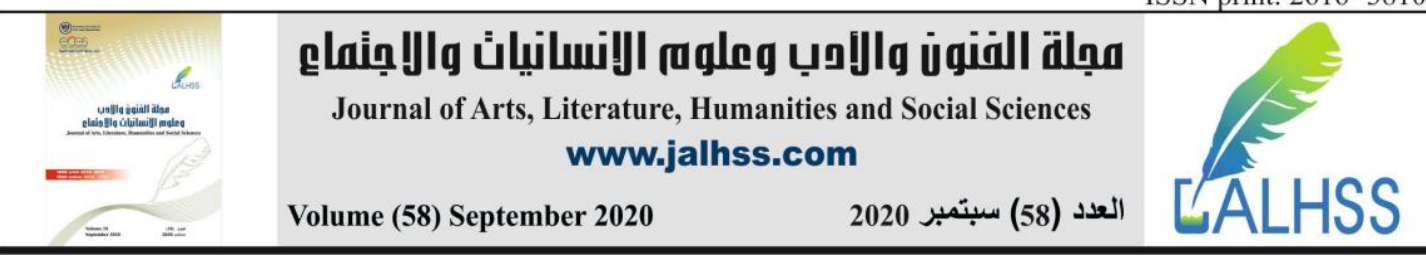

المبحث الثالث

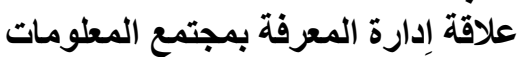

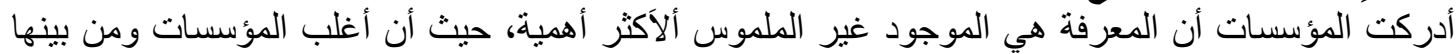

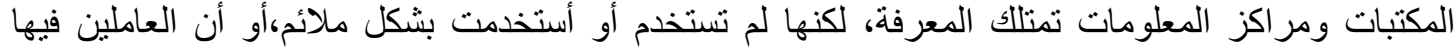

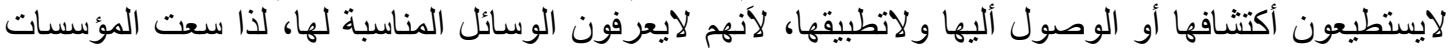

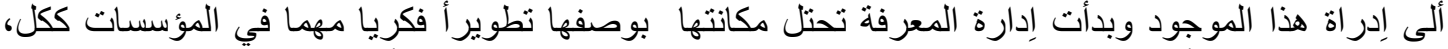

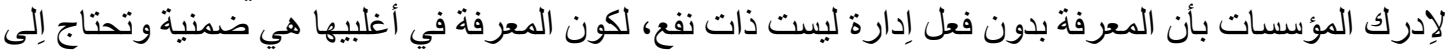

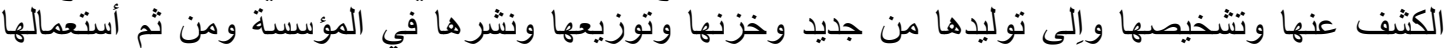

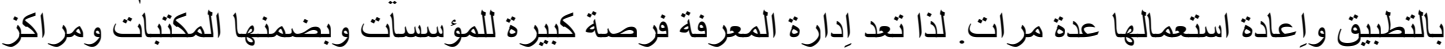

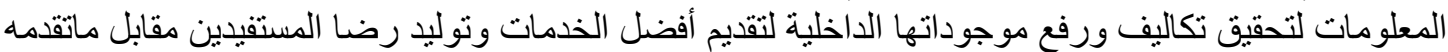

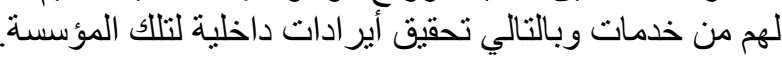

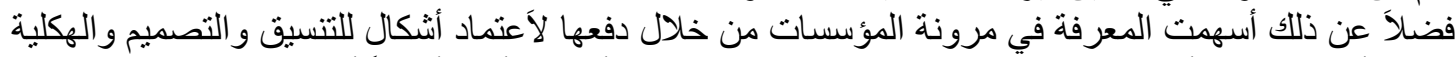

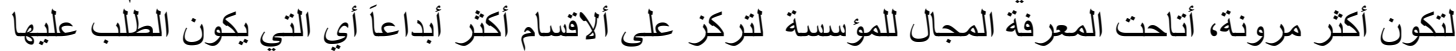

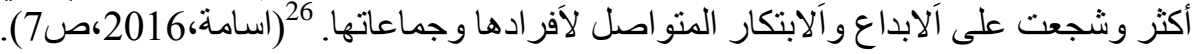

لذا فاَن إدارة المعرفة تلعب دور مهم في دعم المؤسسات من خلال مايلي:

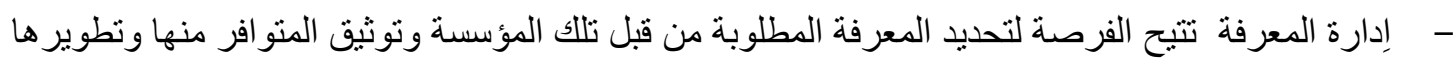
وتطبيقها والمشاركة بيها. - ـ دعم الجهود للاستفادة من جميع الموجودات الملموسة وغير الملموسة وذلك بتوفير إِار عمل لتعزيز

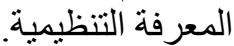
- - من خلال مساهمتها في تعظيم قيمة المعرفة ذاتها عبر التركيز على موجودات المكتبة.

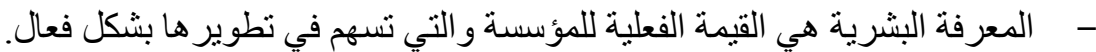

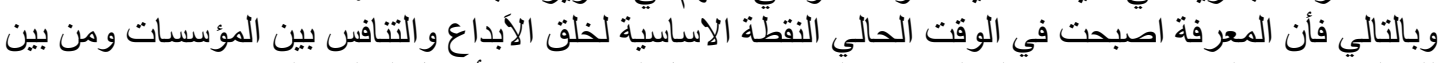
تللك المؤسسات المكتبات ومر اكز المعلومات و المؤسسات ككل لتقديم ما هو أفضل لمتلقي الخدمة.

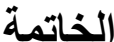

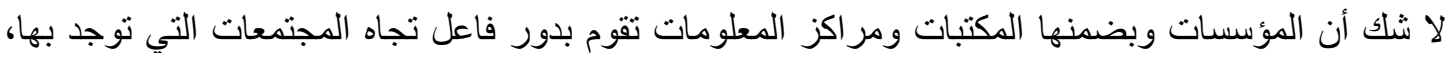

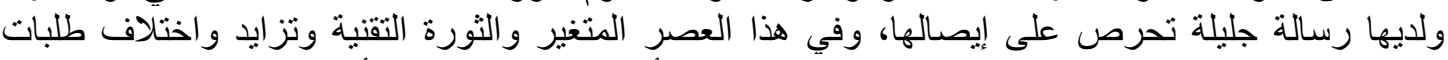

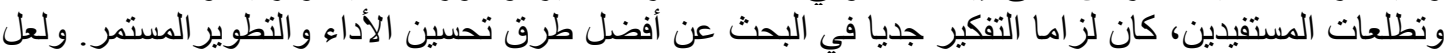

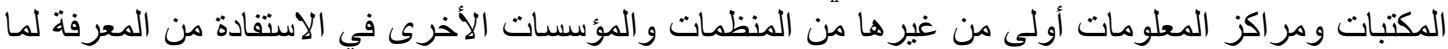

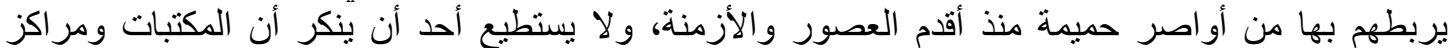

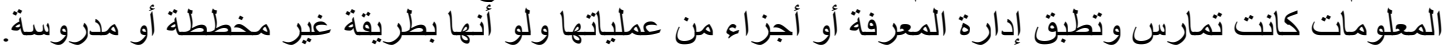

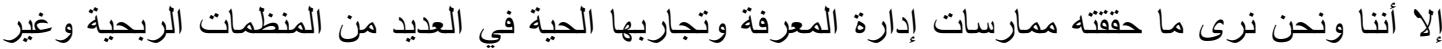

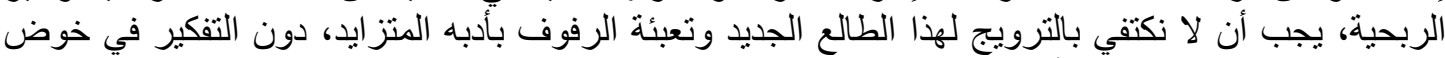

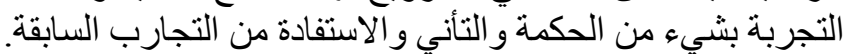




\section{هوالة الفiو

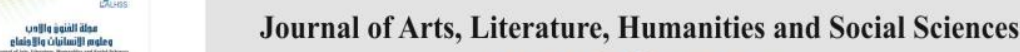 www.jalhss.com \\ 1. .قطر، محمود، أدارة المعرفة أم معرفة ألادارة، الملتقي العربي للأساليب الحديثة للمكتبات .ـ الاسكندرسة : مصر .502005، 2. الكبيسي ،صلاح الدين ،أدارة المعرفة .- القاهرة :المنظمة العربية للتنمية الإِدرية ،2005،صاص11. 3 5 4 .الكبيسي، المصدر السابق نفساه ، ص232. 5. العلي ، عبد الستار، عامر أبراهيم القنديلجي،غسان العمري، المدخل إلي إدارة المعرفة.- عمان : دار الميسرة 260 2006،

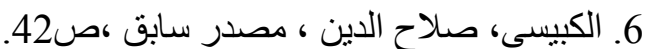

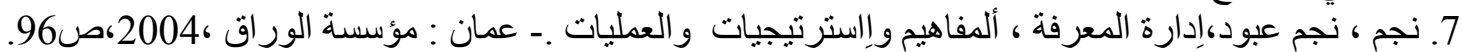

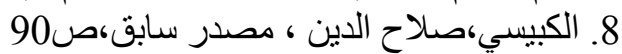

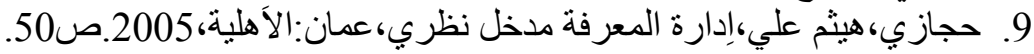

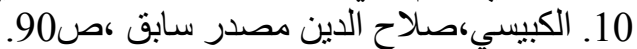

11. Hamid, S. \& Nayan, J. M. .Preliminary study of knowledge management in a library: a case study of the national library of Malaysia. In :international conference on libraries. Palau Pinang: 2005,p17,Available at :http//www.lib.usm.m/elmu-equip<Accessed in $12 / 3 / 2020$.

12. Wen, Sh. . Implementing Knowledge Management in Academic Libraries: A Pragmatic Approach. In : the 3rd China-US Library Conference.. Available a t:http //www.white-clouds.com/iclc,Accessed in 12/3/2020.

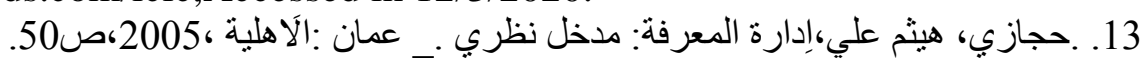

14 .Knowledge Management Section. IFLA Home Page. Available at http//www.ifla.org,Accessed in 12/3/2020.

15. هثمرى ، عمر أحمد ـ الإدارة الحديثة للمكتبات مر اكز المعلومات .ـ-عمان : مؤسسة الرؤي العصرية ،2001 ، ص صن 153

16. عبد الو هاب ، سمير محمد ـ متطلبات تطبيق إدارة المعرفة في المدن العربية ـ در اسة حالة مدينة القاهرة في الندوة

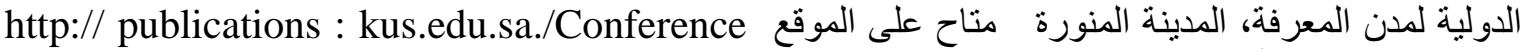
19/7/2020. knowledge.

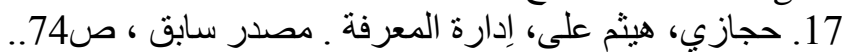

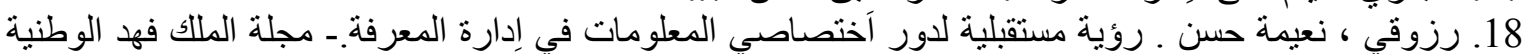

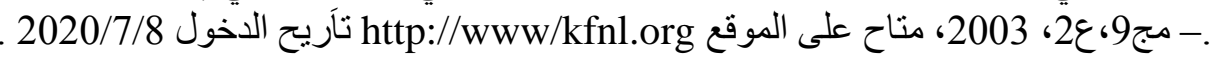

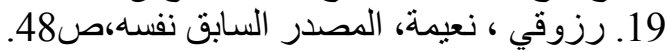

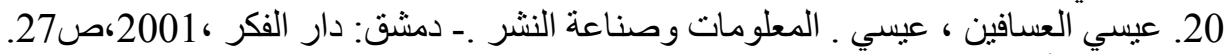

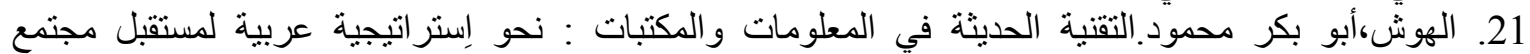

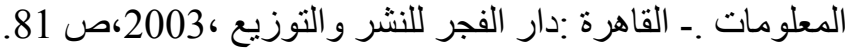

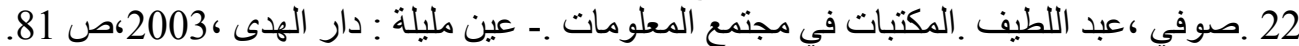
23. حسين ،عبد الرزاق، على الخط المبانشر على htm health ,htt//www بتاريخ 2020/3/18 2002004، 24. كامل شاهينشريف. مصادر المعلومات الإِكترونية في المكتبات ومراكز التوثيق .ـ القاهرة : الدار المصرية

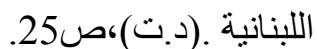

Anne ,M ,Moulin .Les Bibliotheques, Toulouse ,Editions Milan.2014 p33. .25

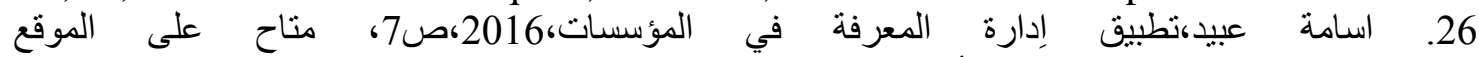

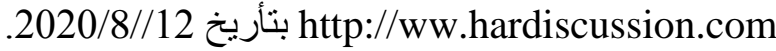




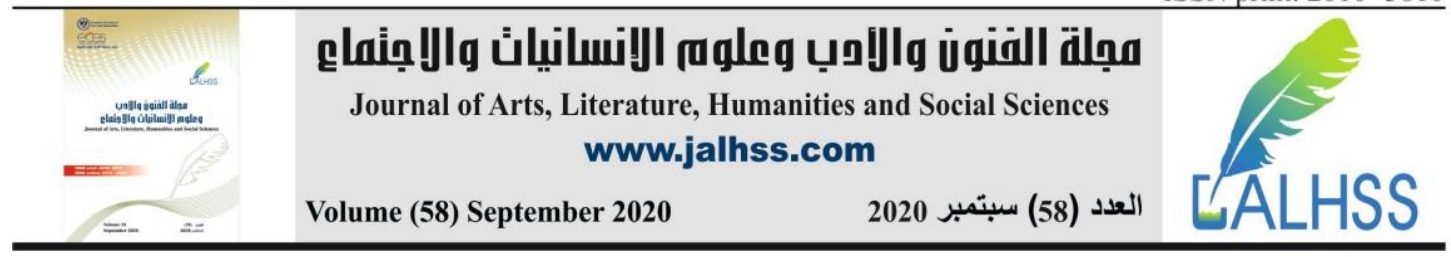

\section{References}

1,Qatar, Mahmoud, Knowledge Management or Knowledge Management, The Arab Forum for Modern Methods of Libraries.

2. Al-Kubaisi, Salah El-Din, Knowledge Management .- Cairo: The Arab Organization for Administrative Development, 2005, p. 11.

3. Al-Kubaisi, Salah .previous source, p. 12.

4. Al-Kubaisi ,Same previous source, p. 32.

5. Al-Ali, Abdul Sattar, Amer Ibrahim Al-Qandilji, Ghassan Al-Omari, Introduction to Knowledge Management - Amman: Dar Al-Maisara, 2006, p. 26.

6. Al-Kubaisi, Salah Al-Din, previous source, p. 42.

7. Najm, Najm Abboud, Knowledge Management, Concepts, Strategies and Operations - Amman: Al-Warraq Foundation, 2004, p. 96.

8. Al-Kubaisi, Salah Al-Din, previous source, p. 90

9. Hijazi, Haitham Ali, Knowledge Management A theoretical introduction, Amman: Al-Ahlia, 2005. p.50.

10. Al-Kubaisi, Salah .previous source, p. 90.

11. Hamid, S. \& Nayan, J. M. .Preliminary study of knowledge management in a library: a case study of the national library of Malaysia. In : international conference on libraries. Palau Pinang: 2005,Available at :http//www.lib.usm.m/elmuequip<Accessed in 12/3/2020.

12.Wen, Sh. . Implementing Knowledge Management in Academic Libraries: A Pragmatic Approach. In : the 3rd China-US Library Conference.. Available a t:http //www.white-clouds.com/iclc,Accessed in 12/3/2020.

13.Hijazi, Haitham Ali, Knowledge Management: A theoretical introduction. Amman: Al-Ahlia, 2005, p.50.

14.Knowledge Management Section. IFLA Home Page. Available at http//www.ifla.org,Accessed in 12/3/2020.

15.Hashmari, Omar Ahmed. Modern Management of Libraries and Information Centers. - Amman: Modern Vision Foundation, 2001, p.153.

16. Abdel-Wahhab, Samir Muhammad. Requirements for applying knowledge management in Arab cities. A case study of Cairo at the International Symposium on Cities of Knowledge, Medina, is available on the website http:// publications: kus.edu.sa./Conference /knowledge. Assessed in 19/7/2020 .

17.Hegazy, Haitham Ali, knowledge management. Previous source, p. 74.

18. Razouki, Naima Hassan. A future vision of the role of the information specialist in knowledge management. - King Fahd National Journal - Volume 9, Issue 2, 2003, Available at http ://www/kfnl.org. Entry permit ,Accessed in 7/8/2020.

19. Razzouki, Naima, The same previous source, p. 48.

20. Issa Al-Assafin, Issa. Information and the publishing industry, Damascus: Dar AlFikr, 2001, p27.

21. Al-Hosh, Abu Bakr

Mahmoud, Modern Information Technology and Libraries: Towards an Arab Strategy for the Future of the Information Society - Cairo: Dar Al-Fajr for Publishing and Distribution, 2003, p. 81. 


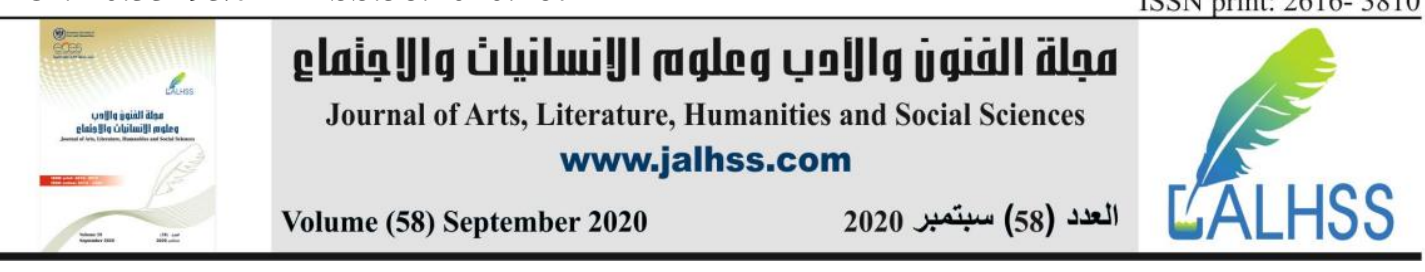

22- Sophie, Abdulateef, Libraries in the Information Society, Ain Melilla: Dar AlHoda, 2003, p. 81.

23 Hussain, Abd Al-Razzaq, online at http://www.health-health/htm on 3/18/2020, 2004, p.20.

24..Kamel Shaheen, Sherif. Sources of electronic information in libraries and documentation centers .- Cairo: The Egyptian Lebanese House (DT), p. 25 .

25. 25.Anne ,M ,Moulin .Les Bibliotheques ,Toulouse ,Editions Milan.2014 p33.

26. Osama Obeid, Application of Knowledge Management in Institutions, 2016, p.7, Available at http://ww.hardiscussion.com ,Accessed in 8/12/2020. 